\title{
Towards high-density recording of brain-wide neural activity
}

\author{
Bo Liang and Xuesong $\mathrm{Ye}^{*}$
}

Brain activity is highly structured within local microcircuits and brain-wide networks, involving exquisite coordination across multiple brain regions in both superficial and deep structures [1]. To understand how brain represents, transforms and communicates information requires simultaneously monitoring distributed neural activity at brain-wide scale with single neuron resolution [2]. Extracellular electrical recording probes can detect spikes from individual neurons within milliseconds, however, their contact site numbers are limited and only a few dozen neurons per probe can be recorded [3,4]. More contacts can be achieved with probe array coupled to external amplification and multiplexing electronics. However, too many transmission wires and large form factor hardware make it impractical to use in freely moving rodents [5]. Optical approaches such as $\mathrm{Ca}^{2+}$ imaging cover multiple areas across spatial scales, but cannot identify the precise timing of spikes of neuronal activity due to their low temporal resolution [2]. Simultaneously monitoring neural activities across multiple brain regions at neuronal scale with high temporal resolution is still challenging.

To meet this challenge, Jun and colleagues [6] have developed a silicon based probe called Neuropixels containing 960 sites on a single shank, which can simultaneously record the activity of more than 200 individual neurons. What's more, the length of the recording shank as long as $10 \mathrm{~mm}$ enables recording from multiple brain regions simultaneously, and even the deepest structures of a mouse brain can be accessed.

The dense and extensive recording sites on a single probe was achieved using a custom $130 \mathrm{~nm}$ CMOS fabrication process. This process allowed Jun and colleagues to place 960 sites on a single shank with $70 \mu \mathrm{m} \times 20 \mu \mathrm{m}$ cross-section. The recording sites are arranged in a checkerboard pattern with 4 columns (Fig. 1a). The authors also integrated low noise analogue amplifiers, multiplexers and digitizers into the probe base (Fig. 1b) with the same CMOS process. According to the cuttingedge electronics, 384 of the 960 total sites on the shank can be addressed simultaneously by user programmable switches, allowing active recording sites to be altered after implantation. Data from all 384 channels coul d be digitized and compressed in the probe base, realizing a digital output stream transmitted via a single thin cable, enabling the device to be compatible with long-term attachment to small animals with natural behaviour.

A key element in the Neuropixels probe is the recording site material. The typical material (PEDOT coated gold [7]) used to make recording contacts is not compatible with the electronic fabrication process of Neuropixels. The authors developed porous titanium nitride (TiN) for the recording site material (Fig. 1c). They found that probes based on TiN performed as well as those made of conventional materials when implanted in animals for 8 weeks. What's more, sites made of TiN are compatible with the CMOS processing, ensuring scalable fabrication of the Neuropixels probes at low cost [6].

The long recording length and abundant recording sites of Neuropixels probes allowed researchers to obtain electrophysiological measurements across a large spatial extent. As an example, the authors inserted a probe into the brain of an awake, head-fixed mouse, targeting the primary visual cortex and the lateral posterior nucleus of the thalamus. Because the probe records activity with the same spatial resolution along the entire shank, the data can be conveniently displayed as images with each site represented as a "pixel". Using these images, structural boundaries can be visualized using simple measures of neural activity, such as firing rates or signal amplitude

College of Biomedical Engineering and Instrument Science, Key Laboratory of Biomedical Engineering of Ministry of Education, Biosensor National Special Laboratory, Zhejiang University, Hangzhou 310027, China

*Email: yexuesong@zju.edu.cn 

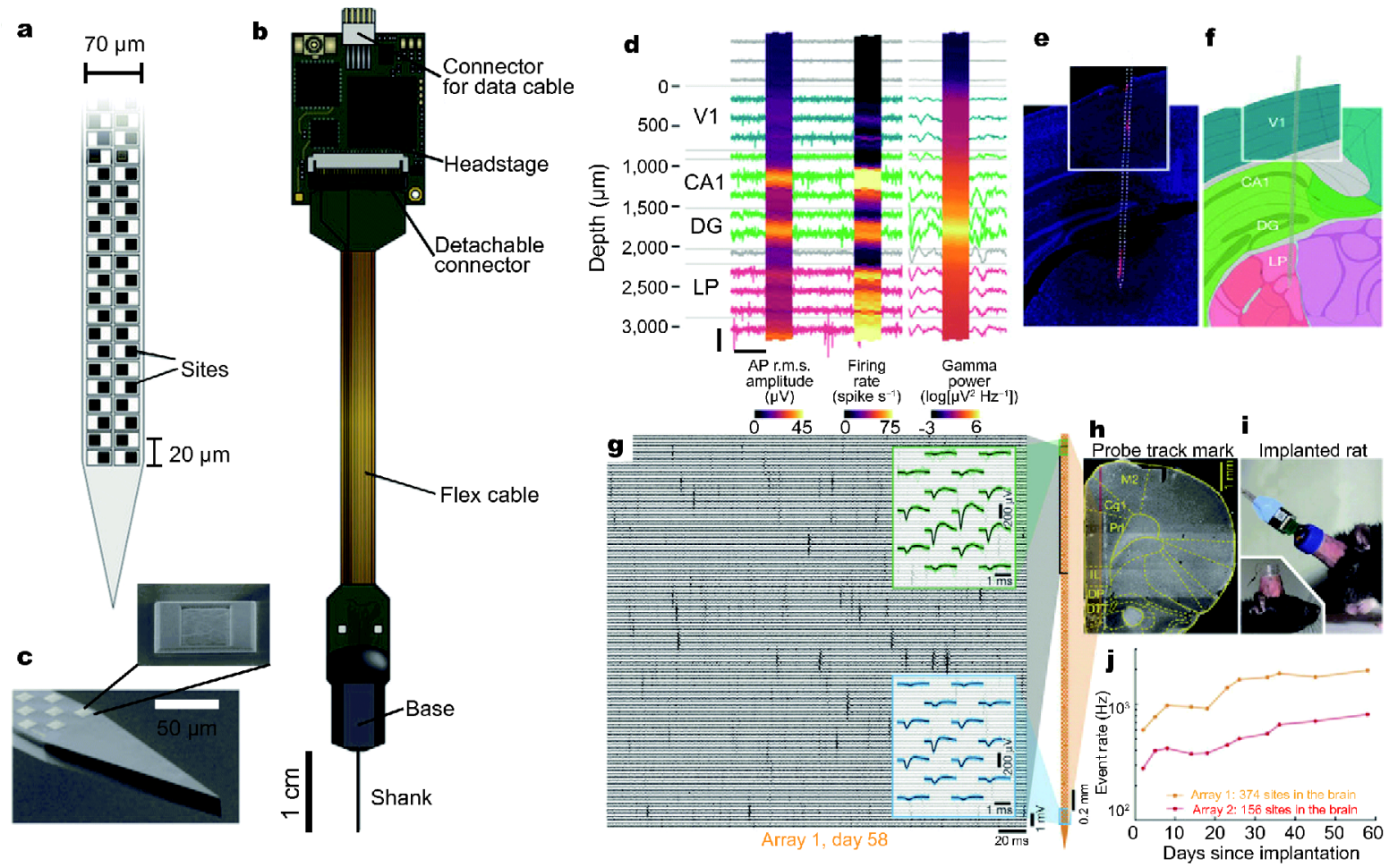

Figure $1(a-c)$ The Neuropixels probe. (a) Illustration of probe tip, showing checkerboard site layout (dark squares). (b) Probe packaging, including flex cable and headstage for bidirectional data transmission. (c) Scanning electron microscopy image of probe tip. (d-f) Recording from large neuronal populations with a single probe in an awake head-fixed mouse. Signals were acquired from a Neuropixels probe inserted through primary visual cortex (V1), CA1 and dentate gyrus (DG) regions of the hippocampus, and the lateral posterior (LP) nucleus of the thalamus. Approximate structure boundaries are shown in grey next to the probe depth scale. (d) For the heat maps, each square represents a single site. Left, r.m.s. amplitude of the AP band signal for 1-s intervals, averaged over 10 intervals. Middle, Firing rate measured from AP band crossings of a $-50 \mu \mathrm{V}$ threshold, in a 10-s interval. Right, Gamma-band LFP power $(35-80 \mathrm{~Hz})$, computed by Welch's method in a 1-s interval, averaged over 10 intervals. Behind the heat maps are example traces from every twenty-fifth channel shown for the AP band (behind panels a and b) and the LFP band (behind panel c), $n=103$ neurons in the thalamus, 41 in the hippocampus, and 62 in the cortex. Vertical/horizontal scale bars: AP band (a, b), $315 \mu \mathrm{V} / 165 \mathrm{~ms} ; \mathrm{LFP}$ band (c), 1,550 $\mu \mathrm{V} / 290$ ms. (e) Histological reconstruction of the probe track with 4',6-diamidino-2-phenylindole (DAPI; blue) and DiI (red) staining. (f) Corresponding images 81 and 82 from Allen Mouse Brain Atlas. (g) mPFC recording ( $n=3$ rats), $200 \mathrm{~ms}$ of voltage traces and examples of waveforms from two sorted neurons. Insets, dark lines show average waveform overlaid with 30 randomly selected single event raw waveforms of a neuron located at the top (green) and bottom (blue) of the distal most group of 384 sites (array 1). (h) Anatomical targeting of the chronic implant in mPFC. Section was immunofluorescently labelled with IBA-1. Cg1, cingulate cortex, area 1; DP, dorsal peduncular cortex; DTT, dorsal tenia tecta; IL, infralimbic cortex; M2, secondary motor cortex; Prl, prelimbic cortex. (i) Photos showing a rat with a chronically implanted Neuropixels probe in the mPFC. (j) Plot of total event rate (summed over 374 sites for array 1 and 156 sites for array 2) across recording sessions over 60 days.

(Fig. 1d-f). In another example experiment, two probes were inserted into a mouse brain. One probe spanned the sensory cortex, hippocampus and thalamus, while the second probe spanned motor cortex and striatum. In this experiment, more than 700 putative single neurons from both superficial and deep structures were recorded simultaneously.

Neuropixels probes are ideal for large scale recordings in freely moving animals owing to their fully integrated functionality and small size. In a chronic implantation experiment, a probe was implanted in the rat medial prefrontal cortex (mPFC), and delivered recordings of hundreds of neurons simultaneously for more than 8 weeks (Fig. 1g-j). Further, light induced artefact can be minimized using sinusoidal or ramped light pulses or subtracted out, allowing the combination use of Neuropixels recordings with optogenetic stimulation in vivo [8].

In summary, Neuropixels probes enables electrical recording from brain-wide regions simultaneously at neuronal scale with high temporal resolution, allowing direct tests of the relationships between connectivity and function across both local and distant brain structures [9]. This technology is an essential step towards understanding the global coordination of activity fundamental 
to brain function and opens a door to exciting experiments aimed at studying functional neuronal circuits.

Received 30 November 2017; accepted 4 December 2017;

published online 8 January 2018

1 Lewis CM, Bosman CA, Fries P. Recording of brain activity across spatial scales. Curr Opin NeuroBiol, 2015, 32: 68-77

2 Harris KD, Quiroga RQ, Freeman J, et al. Improving data quality in neuronal population recordings. Nat Neurosci, 2016, 19: 11651174

3 Buzsáki G, Anastassiou CA, Koch C. The origin of extracellular fields and currents-EEG, ECoG, LFP and spikes. Nat Rev Neurosci, 2012, 13: 407-420

4 Williams JC, Rennaker RL, Kipke DR. Long-term neural recording characteristics of wire microelectrode arrays implanted in cerebral cortex. Brain Res Protocols, 1999, 4: 303-313

5 Shobe JL, Claar LD, Parhami S, et al. Brain activity mapping at multiple scales with silicon microprobes containing 1,024 electrodes. J Neurophysiol, 2015, 114: 2043-2052

6 Jun JJ, Steinmetz NA, Siegle JH, et al. Fully integrated silicon probes for high-density recording of neural activity. Nature, 2017, 551: 232-236

7 Ludwig KA, Langhals NB, Joseph MD, et al. Poly(3,4-ethylenedioxythiophene) (PEDOT) polymer coatings facilitate smaller neural recording electrodes. J Neural Eng, 2011, 8: 014001

8 Anikeeva P, Andalman AS, Witten I, et al. Optetrode: a multichannel readout for optogenetic control in freely moving mice. Nat Neurosci, 2012, 15: 163-170

9 Nicolelis MAL. Opinion: brain-machine interfaces to restore motor function and probe neural circuits. Nat Rev Neurosci, 2003, 4: $417-422$ 